\title{
Effet de fréquence et d'âge d'acquisition dans une tâche de fluence verbale chez des francophones atteints de la maladie d'Alzheimer et des personnes âgées saines
}

\author{
Camille Frouin $^{1}$, Frédérique Gayraud ${ }^{1} \&$ Melissa Barkat-Defradas ${ }^{2}$ \\ ${ }^{1}$ Laboratoire Dynamique Du Langage (UMR 5596), CNRS \& Université de Lyon \\ ${ }^{2}$ Laboratoire Praxiling (UMR 5267), CNRS \& Université Montpellier-III \\ camille.frouin@univ-lyon2.fr \\ frederique.gayraud@univ-lyon2.fr \\ Melissa.Barkat-Defradas@univ-Montp3.fr
}

\begin{abstract}
Résumé. Le but de cette étude est d'observer les effets de fréquence et d'âge d'acquisition dans les productions de sujets francophones atteints de la maladie d'Alzheimer et de sujets âgés sains lors d'exercices requérant la recherche, la récupération et l'activation du lexique en mémoire sémantique. Pour ce faire, nous avons soumis nos sujets à deux tâches de fluence verbale : une sémantique (animaux) et l'autre littérale $(P)$. Les résultats montrent que les sujets atteints de la maladie d'Alzheimer réussissent moins bien que les sujets contrôles la recherche sémantique (vs littérale) et produisent moins de mots que les sujets sains. Leurs productions contiennent également des mots plus fréquents et acquis plus tôt que les mots produits par les sujets sains. Une régression logistique montre que l'âge d'acquisition à lui seulnest capable de prédire le groupe d'appartenance d'un sujet.
\end{abstract}

\section{Introduction}

La maladie d'Alzheimer est une pathologie neurodégénérative responsable d'un déclin cognitif profond et irréversible (Campion, Hannequin, 2002 ; Puel, Démonet, Ousset, Rascol, 1991). Elle représente le type de démences séniles le plus fréquent en France et dans le monde, et sa prévalence ne cesse d'augmenter (Helmer, INSERM, 2004 ; Berr, Tasnime Akbaraly, Fati Nourashemi, Andrieu, 2007). L'un des facteurs qui permet d'expliquer cette augmentation est le vieillissement de la population : aujourd'hui en France, près d'une personne sur cinq a plus de 65ans (INSEE, 2010). En 2035, trois personnes sur dix auront atteint cet âge (Blanpain \& Chardon, 2010). Or, la prévalence de la maladie d'Alzheimer augmente avec l'âge (Berr, Tasnime Akbaraly, Fati Nourashemi, Andrieu, 2007).

Si la maladie est fréquente aujourd'hui, son dépistage est problématique et posé prudemment sous l'appellation de "démence probable de type Alzheimer », au vu, comme le soulignait McKhann en 1984, du nombre d'erreurs diagnostiques (McKhann, Drachman, Folstein, Katzman, Price \& Stadlan, 1984). En effet, la maladie d'Alzheimer présente des caractéristiques visibles seulement par examen post-mortem. Toutefois, de signes des déficits comportementaux peuvent être perceptibles dès les stades précoces de la maladie puisque les lésions cérébrales causées par la démence ont pour conséquence la détérioration des fonctions cognitives. Des tests cognitifs sont alors mis en place dans 
l'optique mettre à jour ces déficits dans les domaines de l'orientation, la mémoire, le langage etc. Dans ces tests, le langage occupe une place importante. En effet, le langage comporte des indices de cette détérioration cognitive tôt dans le cours de la maladie, et est fortement altéré dans ses stades plus avancés (Caredebat et al., 1991 ; Venneri, Forbes-McKay, Shanks, 2005). Du fait de la progression des lésions cérébrales causées par la maladie d'Alzheimer, l'activité cognitive est réduite. Les fonctions nécessitant de telles ressources se trouvent alors déficitaires (Puel, Démonet, Ousset, Rascol, 1991 ; Donnet, Foncin, Habib, 1991). C'est le cas du langage : tant en production qu'en compréhension, le langage demande des processus complexes et coûteux. En outre, la progression de la démence va elle-même toucher des zones cérébrales responsables du langage (Puel, et al., 1991 ; Donnet, Foncin, Habib, 1991). C'est pourquoi le langage montre des perturbations précoces et importantes dans la maladie d'Alzheimer, tant aux niveaux discursif, que pragmatique et morphosyntaxique. Ainsi, le discours devient désorganisé, plus court et parsemé de longues pauses (Lee, Gayraud, Hirsh, Barkat-Défradas, 2011 ; Forbes, Venneri \& Shanks, 2002 ; Ska \& Duong, 2005). Le contenu informationnel véhiculé se fait également plus pauvre (Melvold, Au, Obler, Albert, 1994), les patients perdent leur initiative à entamer un tour de parole, ils ne font plus usage des stratégies de réparation et la prosodie finit par être atypique (Barkat-Defradas, et al, 2008 ; Melvold et al, 1994). Du point de vue morphosyntaxique, les phrases deviennent de plus en plus courtes, de plus en plus simples, de par leur structure grammaticale, mais également à travers la diminution progressive du nombre de propositions enchâssées (Forbes, Venneri \& Shanks, 2002 ; Venneri, Forbes-McKay, Shanks, 2005 ; Kemper, Marquis, Thompson, 2001). Plus tard, les formes morphologiques irrégulières comporteront des erreurs et la phonologie sera finalement altérée également (Barkat-Defradas, Martin, Duarte, Brouillet, 2008). Néanmoins, c'est au niveau lexical que l'on note les premiers changements, les plus remarquables, imputés à un déficit en mémoire sémantique.

\section{Accès lexical et Maladie d'Alzheimer}

La mémoire sémantique est une composante de la mémoire à long terme. Elle représente le lexique mental d'un individu et stocke ses connaissances du monde : les codes, les règles et les symboles nécessaires à la compréhension et à l'appréhension du monde, les relations entre les concepts, que l'individu peut réactiver et manipuler à tout moment (Tulving, 1972, 1976). La mémoire sémantique est indispensable au langage, tant en compréhension qu'en production, puisque c'est elle qui permet d'attribuer une signification - mentale et verbale - à ce qui nous entoure (Belliard, Peron, Lemoal, Golfier, Vanberten \& Vervelletto, 2005). Les tâches permettant d'évaluer le bon fonctionnement de la mémoire sémantique, telles que les tâches de dénomination d'images, d'appariement mot-image, de fluence verbale ou encore de génération de définitions, laissent transparaître un déficit significatif chez les patients atteints de la maladie d'Alzheimer par rapport au sujets âgés sains (Duarte, Jiménez, Syssay, Launay, 2004 ; Forbes-McKay, Ellis, Shanks \& Venneri, 2005 ; Lymperopoulou, Barry, Sakka, 2006). Ce déficit est dû à une difficulté d'accès au lexique, et se traduit initialement par une anomie (Sambuchi, Michel, Bastien, 2005 ; Barkat-Defradas, Gayraud, Cadhilac \& Lee ,2009). En conséquences, les personnes atteintes d'une maladie d'Alzheimer montreront une augmentation des pauses vides et une diminution du nombre de mots dans leurs productions (Barkat-Defradas, Gayraud, Cadhilac \& Lee, 2009 ; Forbes, Venneri \& Shanks, 2002 ; McKay, Forbes \& Veneri, 2005). Pour compenser ce manque du mot, les patients ont recours à des stratégies de production du langage dès les stades précoces de la maladie, telles que les circonlocutions (i.e. utilisation d'une paraphrase pour définir le mot manquant, par exemple, «c'est pour couper» pour «couteau »), les paraphasies sémantiques (i.e. production d'un mot à la place d'un autre par relation sémantique, par exemple « chaussure» pour «pantoufle» ou «pêche» pour «abricot»), ou les termes génériques (e.g. « chose », « machin », « on », « il ») (Barkat-Defradas, Martin, Duarte, Brouillet, 2008 ; Forbes, Venneri \& Shanks, 2002). Dans les stades plus avancés de la maladie d'Alzheimer, le langage des patients se détériore : le discours spontané montre un appauvrissement significatif du vocabulaire. Enfin, le déficit en mémoire de travail touchera également le niveau phonologique : écholalies (i.e. 
répétition de fin de phrases ou mots entendus), palilalies (i.e. répétition successives de syllabes), persévérations (i.e. répétition due aux difficultés d'inhibition) et paraphasies phonémiques (i.e. transformation de phonèmes par addition, suppression, déplacement ou remplacement, par exemple, /palõ/ pour /balõ/) pourront être fréquentes (Appell, Kertesz \& Fisman, 1982, Lee, 2012).

Pourquoi et comment l'information sémantique est-elle de moins en moins accessible? La réponse est partagée entre problème d'accès à la mémoire sémantique d'une part (Pasquier, Lebert, Grymonprez, Petit, 1995; Nebes, 1989), et problème de connexion à l'intérieur même du réseau, d'autre part (Hodges \& Patterson, 1995 ; Forbes-McKay et al, 2005 ; Rosser \& Hodges, 1994 ; Rascovsky, Salmon, Hansen, Thal, Galasko, 2007). Les chercheurs soutenant la première hypothèse proposent que l'effet d'indiçage, souvent observé chez les personnes atteintes de la maladie d'Alzheimer, est la preuve d'un réseau intact mais d'un accès difficile. Pourtant, il est aussi possible que l'indiçage n'ait aucun effet sur ces sujets. C'est l'un des contre-arguments de Hodges \& Patterson (1995) pour qui la «rupture du réseau sémantique » est évidente au vu des erreurs d'appariement, des erreurs de catégorie et des paraphasies sémantiques. Le fait que le sujet puisse produire un nom à la place d'un autre, sémantiquement liés qui plus est, est une preuve que l'accès au réseau est indemne, mais que les connexions dans le réseau sont devenues affaiblies, inefficaces ou erronées.

Les études sur la mémoire sémantique des sujets atteints de la maladie d'Alzheimer, en lien avec l'hypothèse de la « rupture des réseaux sémantiques » (Hodges \& Patterson, 1995), indiquent que les informations intégrées dans les réseaux les plus actifs tout au cours de la vie sont plus faciles à récupérer et donc mieux récupérées. Ainsi, les personnes atteintes de la maladie d'Alzheimer sont sensibles à la longueur des mots, à leur complexité phonologique, au nombre de voisins phonologiques qu'ils ont, ainsi qu'à leur familiarité, typicalité, fréquence et âge d'acquisition (ForbesMcKay et al, 2005 ; Lee, 2012 ; Gayraud \& Barkat-Defradas 2012 ; Ober, Shenaut, 1988 ; Balota et Ferraro, 1996 ; Gainotti, Di Betta \& Silveri, 1996 ; Lymperopoulou, Barry, Sakka ; 2006). Dans cette étude nous nous intéresserons à deux de ces facteurs : la fréquence et l'âge d'acquisition.

La fréquence d'un mot est la fréquence à laquelle ce mot apparaît dans une langue donnée. Elle peut être calculée à partir d'importants corpus, ou bien par estimation (New, Brysbaert, Veronis, Pallier, 2007). De nombreuses recherches ont montré l'existence de l'effet de fréquence et démontré sa fiabilité et sa force par le poids de celle-ci dans la précision et le temps de réaction du sujet face à un mot fréquent versus non fréquent (Oldfield, Wingfield, 1965 ; Keuleers, Brysbaert, New, 2010). Ainsi, tout locuteur est influencé par un effet de fréquence : les tâches de dénomination donnent un temps de réaction plus long pour les mots non fréquents (e.g. microscope) que pour les mots fréquents (e.g. fleur) (Oldfield, Wingfield, 1965). De même, chez les patients Alzheimer, l'effet de fréquence est probant et visible à chaque niveau. Il se retrouve par exemple dans les tâches de fluence verbale, où les mots produits par les patients sont plus fréquents que ceux produits par les sujets sains (ForbesMcKay et al., 2005). Dans les tâches de décision lexicale et de décision de rimes également, la fréquence influence la latence de production et le nombre d'erreurs, à la fois pour les mots de haute et de basse fréquence (Ober, Shenaut, 1988 ; Balota et Ferraro, 1996). Plusieurs explications coexistent : Selon Balota \& Chumbley (1985), l'effet de fréquence n'est pas que le résultat d'un temps de récupération plus long: les mots non fréquents demandent également plus de temps pour la production orale d'un stimulus. Ce serait donc l'articulation en elle-même, mais pas l'encodage, qui allongerait le traitement. En 1994, Jescheniak \& Levelt révisent les données de Balota \& Chumbley (1985) et précisent que l'influence de la fréquence d'un mot sur le traitement lexical se produit au niveau de la récupération du lexème, c'est-à-dire de la forme phonologique du mot. Grâce à cette étude, les chercheurs montrent que l'effet de fréquence des mots est indépendant de l'effet de répétition, de la difficulté à identifier l'objet, ainsi que des difficultés articulatoires. En situant l'effet de fréquence au niveau de la récupération du lexème, les chercheurs postulent alors que l'encodage phonologique d'un mot fréquent sera exécuté plus rapidement que celui d'un mot non fréquent (Levelt, 1999 ; Jescheniak \& Levelt, 1994). Parallèlement, l'effet de fréquence peut s'expliquer par la théorie des « logogènes » (Morton, 1969). Les « logogènes » sont des dispositifs d'activation lexicale définis par le type d'informations qu'ils peuvent recevoir et par la réponse (lexicale) qu'ils peuvent 
activer. Pour pouvoir atteindre le seuil d'activation nécessaire à la sélection d'un item lexical voulu, le « logogène » a besoin d'une quantité d'informations (sémantiques, phonologiques, contextuelles). Or, les «logogènes » responsables de l'activation des mots fréquents, ne retombent pas tout à fait à un degré d'activité nul ; atteindre le seuil d'activation pour eux nécessite donc moins d'informations que pour les mots non fréquents. La latence de dénomination pour les mots fréquents est donc réduite puisque le temps d'activation de ce mot se fait plus rapidement que pour les mots non fréquents; le système le reconnaît plus rapidement.

L'âge d'acquisition est l'âge auquel un mot est acquis pour la première fois. Il représente une variable influente dans la reconnaissance et la récupération des mots en mémoire (Morton, 1969). Tout comme la fréquence, son effet est démontré par les tâches de dénomination d'images par exemple, dans lesquelles les sujets reconnaissent plus rapidement les mots acquis tôt que les mots acquis tard (Caroll \& White, 1973 ; Ellis \& Morrison, 1998). Si l'âge d'acquisition a longtemps été mis de côté car considéré comme simple conséquence de l'effet de fréquence (les mots acquis tôt ne seraient autre que les mots les plus fréquents), il est aujourd'hui source d'intérêt pour les chercheurs dans le domaine de la maladie d'Alzheimer. Plusieurs travaux montrent que l'étude de l'âge d'acquisition dans les productions verbales d'individus est un critère fiable et ce, de manière précoce dans le cours de la pathologie (Forbes-McKay et al., 2005). En effet, les patients donnent des mots acquis plus tôt que les sujets sains dans des tâches de fluence sémantique (Forbes-McKay et al., 2005) et obtiennent de meilleurs résultats (nombre de bonnes réponses) pour les mots acquis tôt en tâche de dénomination d'images (Lymperopoulou, Barry, Sakka, 2006). Pourquoi un tel facteur influencerait de la sorte la récupération du lexique ? En 1984, une étude de Gilhooly s'intéresse au temps de résidence des mots en mémoire. Plus précisément, le chercheur suppose que plus un mot est acquis tôt, plus il passe du temps en mémoire. Ce temps passé en mémoire serait déterminant car rendrait les processus d'amorçage et de récupération plus rapides et plus efficaces. Plus tard, l'Hypothèse de la Fréquence Cumulée, c'est-à-dire, le «nombre total d'expériences relatives à un mot que nous avons au cours de notre existence » (Bonin, 2003, p.125), est énoncée par Morrison, Hirsh, Chappell \& Ellis (2002). Cependant, les recherches montrent que l'effet d'âge d'acquisition à lui seul a plus d'influence (voir aussi Ghyselinck, Lewis \& Brysbaert, 2004). Dans une stratégie différente, Brown \& Watson (1987), privilégient l'hypothèse phonologique. Selon eux, les enfants stockeraient leur lexique sous forme d'unités non segmentées. L'adulte, quant à lui, à cause d'un manque de plasticité cérébrale, a besoin de mettre en place de nouvelles stratégies et stockerait alors ses connaissances lexicales en unités segmentées. Cette façon de procéder, certes plus économique pour le stockage, est plus longue à récupérer et permettrait d'expliquer la différence de traitement pour les mots acquis tôt/tard. En 2000, Ellis \& Lambon Ralph avancent que le lexique acquis tôt dans l'existence bénéficierait d'un réseau renforcé, tel les bases solides d'un savoir, dont ne profiteraient pas les mots acquis tard.

La prise en compte de l'âge d'acquisition nous permettra ici d'évaluer la validité de la théorie de la rétrogenèse (Reisberg, Franssen, Hasan, Monteiro, Boksay, Souren, Kenowsky, Auer, Elahi, Kluger, 1999) appliquée au langage. Depuis les années 1960, les scientifiques ont prouvé une symétrie quasiparfaite entre le développement normal de l'humain et la perte de ses fonctions cognitives, mentales, fonctionnelles et biologiques dans les démences séniles (Riesberg, Franssen, Souren, Auer, Akram, Kenowsky, 2002). Reisberg et son équipe nomment cette inversion la théorie de la rétrogenèse et la définissent comme étant le processus par lequel «les mécanismes de la démence inversent l'ordre d'acquisition du développement normal » (Reisberg et al., 1999, p.28). Les facultés développées le plus tardivement chez l'enfant, seront altérées le plus précocement chez le patient Alzheimer (Reisberg et al., 1999). Ce phénomène touche tous les niveaux comportementaux (Rogers, Lasprilla, 2006) mais ne compte pas encore de nombreux travaux relatifs à la rétrogenèse du langage. L'étude de l'âge d'acquisition des mots ici devient alors pertinente.

Dans une tâche de fluence verbale, nous comparerons les productions de patients atteints de la maladie d'Alzheimer avec celles de sujets âgés sains, afin d'en analyser la fréquence et l'âge d'acquisition. 
Les mots fréquents étant plus faciles à récupérer, nous supposons que les sujets atteints de la maladie d'Alzheimer les produiront préférentiellement.

Si l'hypothèse de la rétrogenèse (Reisberg et al., 1999) s'applique au langage, les patients Alzheimer devraient produire des mots acquis plus précocement dans l'existence que les participants contrôles.

Suivant Forbes McKay et al. (2005), nous nous attendons à ce que ces deux facteurs soient corrélés entre eux.

Enfin et étant donné que les patients Alzheimer présentent un déficit sémantique, ils devraient produire moins de mots dans la tâche de fluence sémantique que dans la tâche de fluence littérale.

\section{Méthodologie}

\subsection{Sujets}

20 sujets atteints d'une Démence de Type Alzheimer (MA) (19 femmes et 1 homme) sur la base du NINCDS ADRDA, (McKhann et al., 1984) et 20 sujets âgés sains (11 femmes, 9 hommes), appariés en âge et niveau d'étude (en nombre d'années) étaient volontaires pour cette étude. Selon le MMSE (Folstein et al., 1975) le degré d'avancement de la démence pour notre groupe de patients va de la démence légère à la démence sévère. Tous les participants de l'étude sont droitiers et locuteurs natifs du français. Le tableau 1 illustre nos échantillons de participants.

Tableau 1 : Profil des participants : âge, niveau socio-culturel et score au MMSE.

\begin{tabular}{lcccccccc}
\hline \multicolumn{3}{c}{ Contrôles $(\mathrm{n}=20)$} & \multicolumn{7}{c}{ MA (n=20) } \\
\hline Aoy & ET & Min & Max & Moy & ET & Min & Max \\
Années & 81,1 & 8,1 & 69 & 97 & 84,65 & 13,15 & 70 & 96 \\
d'étude & 11,1 & 3,56 & 6 & 18 & 11,8 & 3,59 & 6 & 19 \\
MMSE & 28 & 1,22 & 26 & 30 & 15 & 4,79 & 5 & 24 \\
\hline
\end{tabular}

Les sujets ne diffèrent ni en âge ni en niveau d'étude; leurs résultats au MMSE sont cependant différents $(\mathrm{p}<0,0001)$.

\subsection{Procédure}

Le recueil des données s'est fait à partir de deux tâches de fluence verbale : une fluence sémantique (animaux) et une fluence littérale (P). Selon le protocole de Cardebat (Cardebat, Doyon, Puel, Goulet, Joanette, 1990), nous avons sélectionné la catégorie sémantique des animaux et la lettre P étant donné que ce sont les deux catégories (sémantiques et littérales) qui offrent le plus grand nombre d'items disponibles en français. Ainsi, nous pensons pouvoir faire ressurgir plus de différences entre les deux groupes. Toujours selon le protocole de Cardebat et al., 1990 (Cardebat, Doyon, Puel, Goulet, Joanette, 1990), chaque sujet disposait d'une minute pour produire le plus grand nombre d'items possible. L'ordre de ces deux tâches était contrebalancé. Les productions étaient enregistrées sur microphone puis transcrites pour analyses.

\subsection{Données}

Toutes les productions des sujets ont été transcrites. Pour chaque production recevable (les persévérations n'ont été comptées qu'une seule fois, tout comme le morphème de base lors de dérivations lexicales; les noms propres et les erreurs ont été supprimés) nous avons recherché une 
fréquence d'occurrence dans la langue (à partir de la base Lexique 3.80 (New, Pallier, Ferrand \& Matos, 2001)) et fait estimer l'âge d'acquisition.

\section{Résultats}

\subsection{Estimations d'âge d'acquisition}

L'estimation de l'âge d'acquisition donne une valeur dite subjective car elle est récupérée à partir d'estimations de sujets adultes, alors que les mesures d'âge d'acquisition objectives sont directement recueillies auprès d'enfants. Toutefois, ces deux sortes de recueil sont très fortement corrélées et nous permettent d'avoir recours aux estimations subjectives de manière fiable et plus économique (Ferrand, Bonin, Méot, Augustinova, New, Pallier, Brysbaert, 2008 ; Chalard, Bonin, Méot, Boyer, Fayol, 2003).

Un groupe de 60 sujets âgés sains ( 23 hommes, 37 femmes) était volontaire ici. Ces sujets étaient tous nouveaux à l'étude, aucun n'avait participé aux tâches de fluence verbale. Une liste de 364 mots, représentant la totalité des productions des sujets en tâche de fluence, leur était présentée. Chaque item attendait une estimation de l'âge auquel il a été appris pour la première fois, aussi bien sous sa forme orale, qu'écrite (sur une échelle allant de 0-2 ans à 13 ans et plus) (Bonin, 2003).

Le choix de l'âge des sujets ici est très important: un effet de génération ressort des estimations. Après avoir recueilli nos estimations auprès de personnes âgées (âge moy $=72,3$ ans), les mêmes estimations ont été menées auprès d'un public jeune cette fois-ci (âge moy $=19,76$ ans). D'après le $t$ test, les jeunes déclarent avoir, en moyenne, acquis les mots testés plus tôt que les personnes âgées (e.g. guépard : AoA jeunes $=5,5$ ans ; AoA âgés $=10$ ans ; $(t(65)=-8,41 ; \mathrm{p}<0,0001))$. Il est difficile de déterminer dans quelle mesure certains mots ont effectivement été acquis plus tard par les personnes âgées. Bien que ce soit vraisemblablement le cas pour certains concepts comme les fruits exotiques (e.g. ananas AoA jeunes $=5,43$ ans ; AoA âgés $=10,28$ ans $(t(65)=-8,42 ; p<0,0001)$. Par exemple lors de la présentation de l'image d'un ananas, l'un des participants de notre étude a dit: « oh bah alors ça, 12 ans et plus hein. Parce que le premier ananas que j'ai mangé c'est en voyage de noces. Je savais pas que ça existait. »). La différence entre les estimations effectuées par des personnes jeunes vs. âgées peut également être due fait de la distance temporelle : les personnes âgées feraient des estimations moins précises que les jeunes. Cependant, rien ne nous permettant de trancher en faveur de l'une ou l'autre de ces explications, nous choisissons d'utiliser les estimations effectuées par des personnes du même âge que nos participants.

Ainsi avons-nous recueilli l'âge d'acquisition de chaque item produit dans la tâche de fluence, par un groupe appartenant à la même génération. Le graphique 1 illustre cette différence d'estimation liée à la génération. 
Graphique 1 : AoA estimés par les sujets jeunes et âgés.

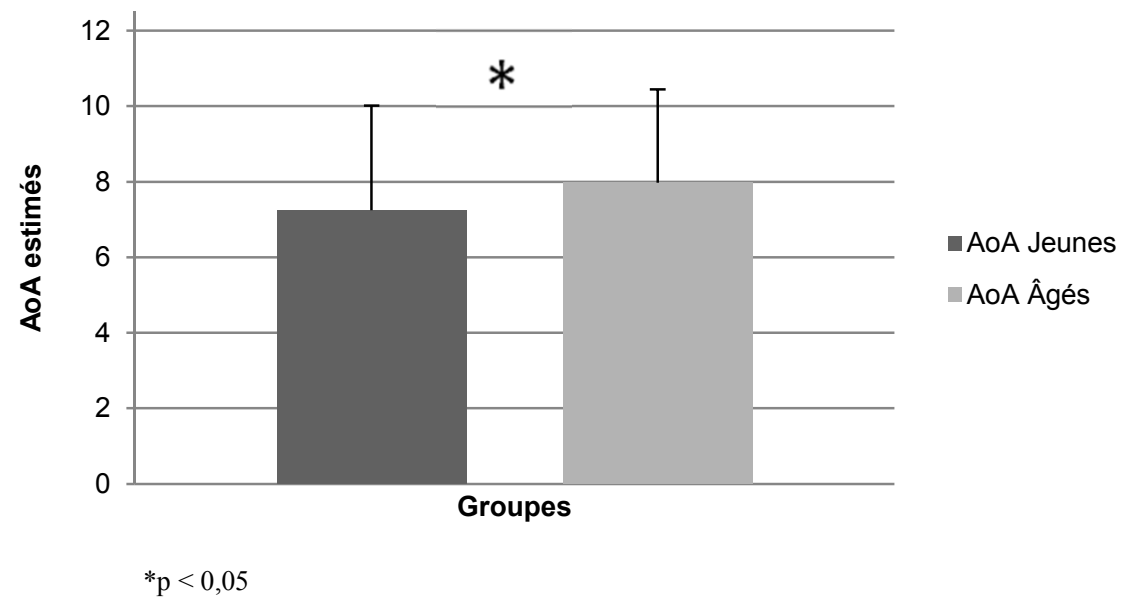

\subsection{Nombre de mots}

L'analyse du nombre de mots produits (persévérations, dérivations et erreurs exclues) par les deux groupes de sujets en tâche de fluence, soumis à une ANOVA, révèle un effet de groupe : les patients produisent moins de mots que les sujets contrôles $\left(F_{(1,38)}=36,41 ; \mathrm{p}<0,0001\right)$. Le test montre également un effet de la tâche $\left(F_{(1,38)}=43,21 ; \mathrm{p}<0,0001\right)$ indiquant que la tâche sémantique donne plus de réponses et l'interaction est significative $\left(F_{(1,38)}=11,8 ; \mathrm{p}=0,001\right)$. Les changements de modalité de la tâche font varier les performances au sein des groupes. Le nombre de mots produits décline donc en fonction de la tâche. Le graphique 2 illustre le nombre moyen de bonnes réponses par groupe et par tâche.

Graphique 2 : Nombre moyen de bonnes réponses par groupe et par tâche.

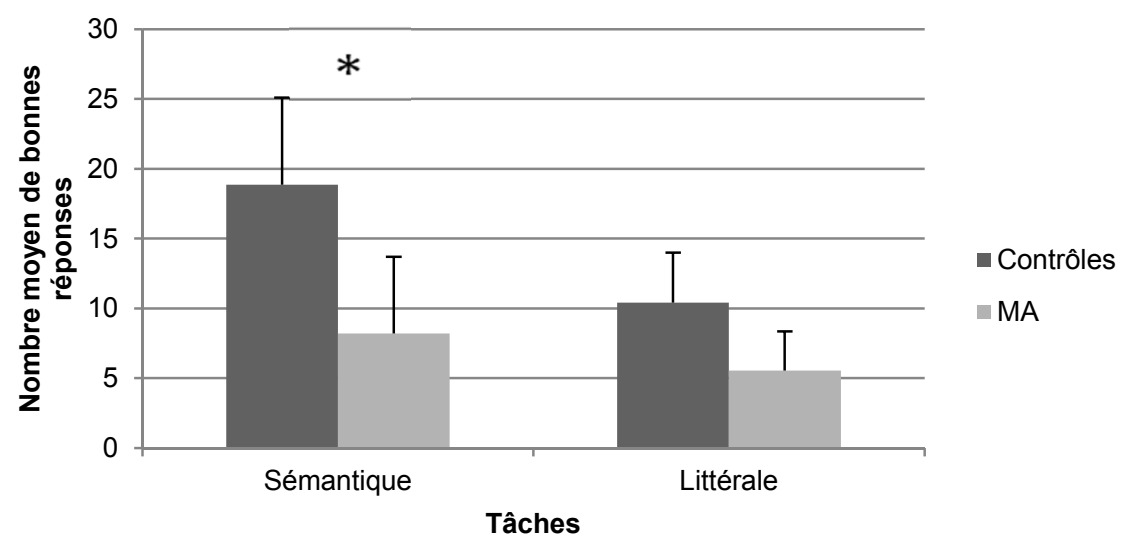

$* \mathrm{p}<0,05$

\subsection{Effet de fréquence}

Nous prenons en compte la fréquence des mots par million d'occurrences; cependant, pour une meilleure représentation de nos analyses, nous avons converti les valeurs de fréquence par une formule logarithmique. En effet, plusieurs études (Adelman, Brown \& Quesada, 2006 ; Segui, Mehler, 
Frauenfelder \& Morton, 1982) remplacent la fréquence par une freq log qui normalise les valeurs. L'intérêt supplémentaire d'une telle formule est qu'elle permet de comparer entre elles, des valeurs très différentes, en réduisant les intervalles. Toutefois, cette formule ne peut s'appliquer qu'à des nombres supérieurs à 0 . Or, nous avons, dans notre corpus, des fréquences dont la valeur est 0 (e.g. " rouge-queue »). Pour obtenir leur freq log, nous avons, comme dans la méthodologie de Adelman et al. [59] remplacé 0 par la valeur de fréquence la plus petite de notre corpus, soit 0,01 . Le graphique 3 représente les freq log moyennes des productions, par groupe et par tâche.

Graphique 3 : Fréquence (freq $\log$ ) moyenne des mots en fonction de la tâche (sémantique vs littérale) et du groupe.

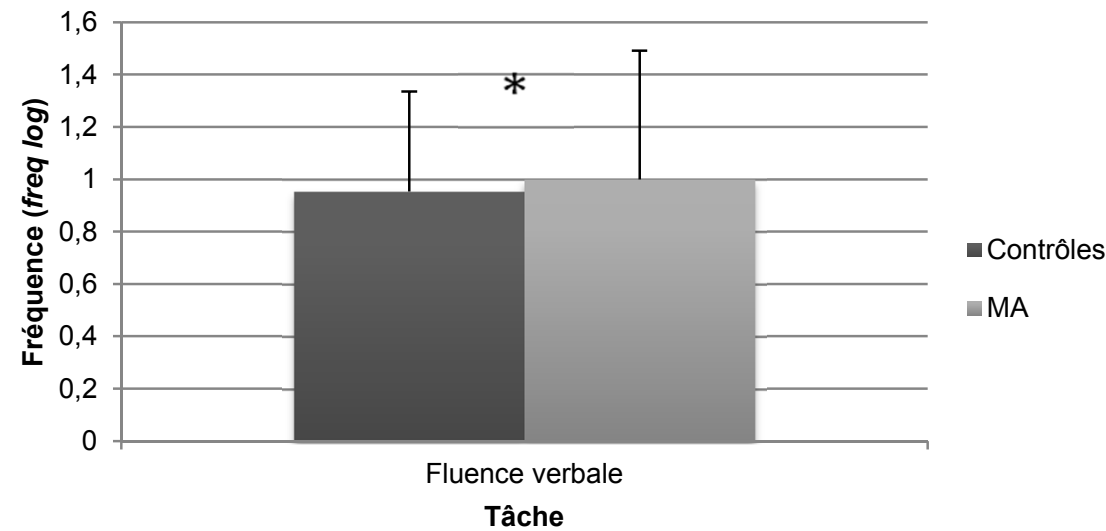

$* \mathrm{p}<0,05$

L'ANOVA appliquée aux valeurs de fréquence d'occurrence des mots produits révèle un effet de groupe : les MA produisent des mots plus fréquents que les sujets contrôles $\left(F_{(1,74)}=25,63 ; \mathrm{p}<\right.$ 0,0001). Cependant, aucun effet de tâche ne ressort ici. De même, aucune interaction entre le groupe et la tâche ne ressort ici. les analyses ne laissent observer aucune interaction.

\subsection{Effet d'âge d'acquisition}

Nous comparons ici l'âge d'acquisition (évalué par des personnes âgées) des mots produits par les deux groupes de sujets. Les résultats montrent un effet de groupe : les patients produisent des mots acquis plus tôt que les sujets contrôles $\left(F_{(1,37)}=11,93 ; \mathrm{p}=0,001\right)$. De même, nous observons un fort effet de tâche : les sujets MA produisent des mots acquis plus tôt en tâche de fluence sémantique $\left(F_{(1,37)}=65,89 ; \mathrm{p}<0,0001\right)$. L'interaction entre les variables tâches et groupes s'approche du seuil significatif. Tous les sujets ont donc tendance à produire des mots acquis plus tôt en tâche sémantique qu'en tâche littérale. Le graphique 4 illustre que cette difficulté en tâche sémantique est d'autant plus vraie pour les sujets MA. Cependant, nous avons observé la même chose pour les sujets sains qui produisent donc des mots acquis plut tôt en tâche sémantique que littérale $(\mathrm{W}(19)=23 ; \mathrm{p}<0,001)$. Le graphique 4 illustre l'âge d'acquisition des mots produits par les sujets dans les deux tâches. 
Graphique 4 : Moyenne d'âge d'acquisition des mots produits, par groupe et par tâche.

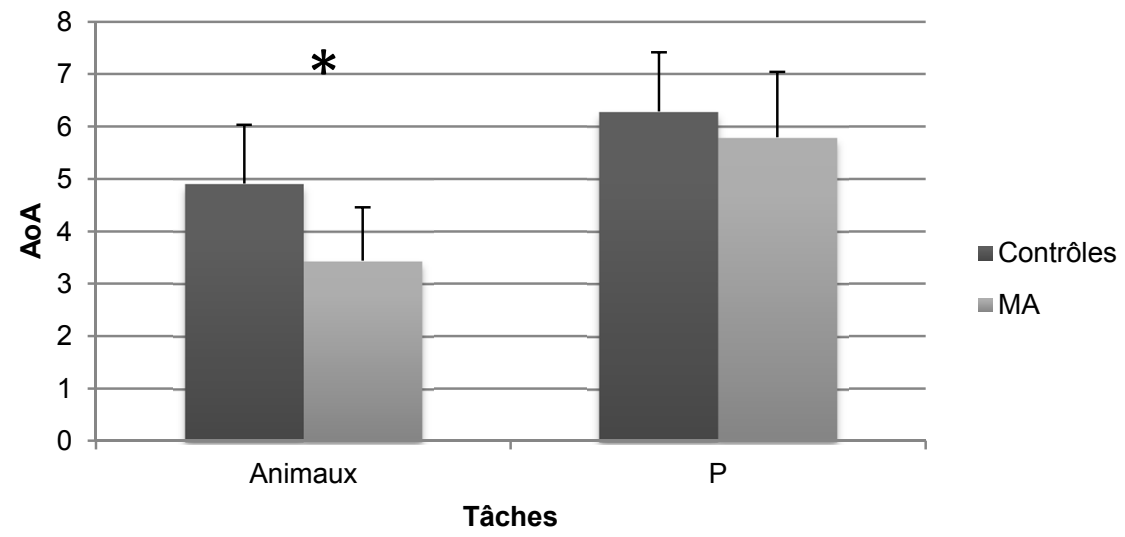

$* \mathrm{p}<0,05$

\subsection{Analyses de corrélations}

Un test de corrélation de Pearson révèle que l'âge d'acquisition et la freq log des productions de tous les sujets et dans toutes les tâches sont négativement corrélés (coefficient de corrélation : $r=-0,62$ ) et ce de manière très significative $(\mathrm{r}(324)=-14,4 ; \mathrm{p}<0,0001)$. De manière générale, toutes tâches et tous sujets confondus, la fréquence et l'âge d'acquisition sont liés; plus la fréquence d'occurrence d'un mot est grande, plus l'âge d'acquisition de ce mot est bas. Le tableau 2 représente la matrice de corrélation.

Tableau 2 : Matrice de corrélations de la fréquence (freq log) et de l'âge d'acquisition (AoA), pour les productions en fluence verbale de tous les sujets.

\begin{tabular}{l}
\hline \multicolumn{2}{c}{ freq log AoA } \\
\hline freq $\log 1$ \\
\hline AoA $-0,62 * * *$ \\
\hline$* \mathrm{p}<0,05 * * \mathrm{p}<0,01 * * * \mathrm{p}<0,0001$
\end{tabular}

Un test de corrélation de Pearson révèle que le poids de la fréquence et de l'âge d'acquisition dans le processus de recherche lexicale diffère selon les sujets et les tâches :

La fréquence (freq $\log$ ) et l'âge d'acquisition sont fortement corrélés dans la tâche sémantique pour les deux groupes de sujets (coefficient contrôles : $r=-0,76$; coefficient Alzheimer : $r=-0,75$ ) et ce, de manière très significative (contrôles : $r(120)=-12,73 ; p<0,0001) ;($ Alzheimer : $r(57)=-8,61 ; p$ $<0,0001)$. Le tableau 3 illustre les matrices de corrélations des deux groupes en tâche sémantique.

Tableau 3(a) : Matrice de corrélations des sujets contrôles en tâche de fluence sémantique

\begin{tabular}{ccc}
\hline & freq $\log$ & AoA \\
\hline freq $\log$ & 1 & \\
\hline AoA & $-0,76^{* * *}$ & 1 \\
\hline $\mathrm{p}<0,05^{* *} \mathrm{p}<0,011^{* * *} \mathrm{p}<0,0001$ &
\end{tabular}

Tableau 3(b): Matrice de corrélations des patients en tâche de fluence sémantique

\begin{tabular}{ccc}
\hline & freq $\log$ & AoA \\
\hline freq $\log$ & 1 & \\
\hline AoA & $-0,75^{* * *}$ & 1 \\
\hline $\mathrm{p}<0,05^{* *} \mathrm{p}<0,01{ }^{* * *} \mathrm{p}<0,0001$ &
\end{tabular}

Toutefois, la corrélation de la fréquence (freq $\log$ ) et de l'âge d'acquisition en tâche littérale montre que, bien que ces deux variables covarient toujours négativement entre elles et de manière très proche dans les deux groupes, les coefficients de corrélation sont moins élevés qu'en tâche sémantique (contrôles : $\mathrm{r}=-0,47$; Alzheimer : $\mathrm{r}=-0.49$ ). (Alzheimer : $\mathrm{r}(47)=-3,81 ; \mathrm{p}=0,0004$; sujets 
contrôles : $\mathrm{r}(94)=-5,13 ; \mathrm{p}<0,0001)$. Le tableau 4 illustre les matrices des deux groupes en tâche littérale.

Tableau 4(a): Matrice de corrélations des sujets contrôles en tâche de fluence littérale.

\begin{tabular}{ll}
\hline & freq $\log$ AoA \\
\hline freq $\log \quad 1$ \\
\hline AoA $\quad-0,47^{* * *} 1$ \\
\hline${ }^{*} \mathrm{p}<0,05^{* * \mathrm{p}}<0,01^{* * * \mathrm{p}}<0,0001$
\end{tabular}

Tableau 4(b): Matrice de corrélations des patients en tâche de fluence littérale.

\begin{tabular}{|c|c|c|}
\hline & freq log & AoA \\
\hline freq log & 1 & \\
\hline AoA & $-0,49 * *$ & 1 \\
\hline
\end{tabular}

\subsection{Analyses de régression logistique :}

La fréquence et l'âge d'acquisition sont deux variables très présentes dans le choix des mots des sujets Alzheimer. Nous avons vu des différences de groupe (sujets contrôles vs sujets Alzheimer) lorsqu'il s'agissait d'analyser la fréquence du lexique ou l'âge d'acquisition des mots produits par tous les sujets. Nous avons également mis en avant une corrélation significative entre ces deux variables pour les deux populations. Ces deux variables semblent donc fortement liées et il est difficile de savoir si ce sont les mots fréquents qui sont acquis tôt, ou si ce sont les mots acquis tôt qui sont fréquents, ou bien encore si ces deux variables ne sont pas dissociables. Nous avons alors séparé le corpus en mots fréquents (i.e. fréquence $>20$ (Lexique, 2003)) puis en mots rares (fréquence $<20$ ), mais également en mots acquis tôt (âge d'acquisition $<6$ ans) ou acquis tard (âge d'acquisition $>6$ ans). Le contrôle de la fréquence ne donne aucun effet de l'âge d'acquisition (e.g. Résultats pour groupe quand les mots ont une fréquence $<20: F_{(1,34)}=0,48 ; \mathrm{p}=0,49$; Résultats pour groupe quand les mots ont une fréquence $>20: F_{(1,37)}=1,4 ; \mathrm{p}=0,24$.), tout comme le contrôle de l'âge d'acquisition ne donne pas d'effet de fréquence (e.g. Résultats pour groupe quand les mots ont un âge d'acquisition $<6$ ans : $F_{(1,37)}=0,66 ; \mathrm{p}=0,42$; Résultats pour groupe quand les mots ont un âge d'acquisition $>6$ ans : $\left.F_{(1,34)}=0,29 ; \mathrm{p}=0,6\right)$. Autrement dit, l'analyse statistique révèle que lorsque nous sélectionnons les mots fréquents du corpus, aucune différence d'âge d'acquisition ne ressort. Il en est de même quand nous sélectionnons les mots rares du corpus. Pareillement, lorsque nous ne gardons que les mots acquis tôt du corpus, la fréquence ne varie pas; elle ne varie pas non plus lorsque nous ne prenons que les mots acquis tardivement.

Pourtant, pour la suite des études, il est important de savoir si ces deux facteurs sont indépendants l'un de l'autre, afin de savoir lequel compte le plus, et par la suite, lequel peut être associé à la maladie d'Alzheimer.

Nous avons donc effectué une analyse de régression logistique dans laquelle nous tentons de prédire l'appartenance au groupe des sujets Alzheimer par l'une des deux variables: fréquence ou âge d'acquisition. Nos résultats indiquent que l'âge d'acquisition ressort comme variable significativement explicative de l'appartenance au groupe des sujets Alzheimer $(\mathrm{p}=0.005)$. La fréquence quant à elle, n'est pas significative. L'âge d'acquisition des mots produits par un sujet semble donc être capable de prédire si ce sujet est un sujet sain ou Alzheimer. Par ailleurs, nous notons que le coefficient de régression pour l'âge d'acquisition est négatif $(r=-0.48)$, confirmant l'idée que plus un sujet produit des mots acquis tôt, plus il a de chances d'appartenir au groupe Alzheimer. Le tableau 5 illustre les résultats de la régression.

Tableau 5: Coefficients de régression, déviation standard, valeur de $\mathrm{z}$ et valeur de $\mathrm{p}$ exprimant la relation entre la production de mots acquis tôt et l'appartenance au groupe des sujets Alzheimer.

\begin{tabular}{lllll}
\hline Attribute & Coefficient & Std-dev & $\mathrm{z}$-value & $\mathrm{p}$-value \\
\hline AoA & $-0,47 * * *$ & 0,169564 & $-2,811$ & $0,00495^{* *}$ \\
\hline Fréquence & 0,001276 & 0,001044 & 1,22 & 0,22159 \\
\hline${ }^{*} \mathrm{p}<0,05 * * \mathrm{p}<0,01 * * \mathrm{p}<0,0001$ & & &
\end{tabular}

D'autre part, la matrice de confusion indique le nombre de sujets bien et mal classés par le modèle, sur la seule base de l'âge d'acquisition. Ainsi, nous pouvons calculer le taux d'erreur (35\%) et le calcul de 
précision, qui annonce qu'un sujet classé comme Alzheimer par le modèle, sur la base de l'âge d'acquisition a $65 \%$ de chances d'être bien classé. De part ces valeurs, et en prenant en compte le rapport de vraisemblance $(11,27)$, nous pouvons estimer le modèle comme étant plutôt fiable.

Tableau 6 : Matrice de confusion :

\begin{tabular}{llll}
\hline & MA & Contrôles & Total \\
\hline MA & 26 & 14 & 40 \\
\hline Contrôles & 14 & 25 & 39 \\
\hline Total & 40 & & 79 \\
\hline
\end{tabular}

Enfin, le calcul de l'odds-ratio indique qu'un sujet qui produit des mots acquis tôt a environ 3 fois plus de chances d'appartenir au groupe des sujets Alzheimer qu'un sujet qui produit des mots acquis plus tard $(\mathrm{OR}=3,32)$. Le tableau 7 illustre ces résultats.

Tableau 7 : Taux d'erreur du modèle, Nagelkerke $\mathrm{R}^{2}$, Odds-ratios, le rapport de vraisemblance et la valeur de $p$ exprimant la relation entre la production de mots acquis tôt et l'appartenance au groupe des sujets Alzheimer.

\begin{tabular}{ccccc}
\hline Taux d'erreur & NagelkerkeR2 & OR & Rapport de vraisemblance & $\mathrm{p}$-value \\
\hline 0,35 & 0,17 & 3,32 & 11,27 & $\mathrm{P}=0,004 * *$ \\
\hline${ }^{*} \mathrm{p}<0,05{ }^{* *} \mathrm{p}<0,01{ }^{* * *} \mathrm{p}<0,0001$ & & &
\end{tabular}

\section{Discussion}

Dans cette étude, nous avons soumis un groupe de patients atteints de la maladie d'Alzheimer à deux tâches de fluence verbale ; l'une sémantique (animaux) et l'autre littérale (P). Afin d'examiner un potentiel effet de fréquence et d'âge d'acquisition sur les productions des patients, un groupe de sujets âgés sains, soumis aux mêmes tâches, faisait office de groupe contrôle.

Nos résultats révèlent que les patients produisent moins de mots que les sujets sains et ce, d'autant plus quand la tâche est sémantique. En effet, les deux groupes produisent plus de mots en tâche sémantique que littérale mais seule la tâche sémantique montre une différence significative entre sujets contrôles et sujets Alzheimer. Comme annoncé dans la littérature, il semblerait que les patients soient moins mis en difficulté dans les recherches phonologiques que sémantiques (Pasquier et al., 1995 ; Cardebat et al.,1990 ; Rascovsky, Salmon, Hansen, Thal, Galasko, 2007). Les sujets sains réussissent significativement mieux les tâches sémantiques puisqu'il serait plus facile et plus rapide d'activer un exemple prototypique (par exemple, «chat » ou «chien » pour la catégorie des animaux) (Rosser, Hodges, 1994) puis d'en activer les voisins proches dans le réseau, plutôt que de n'avoir comme indice qu'une lettre initiale ne nécessitant alors plus une stratégie sémantique, mais phonologique. Cette dernière est alors beaucoup plus vaste et moins orientée donc, moins rapide.

D'autre part, les analyses du corpus révèlent un effet de fréquence entre les groupes. En lien avec les travaux antérieurs, nos hypothèses sont validées (Marczinski \& Kertesz, 2006 ; Forbes-McKay et al., 2005, Kremin et al.,2001; Lymperopoulou et al., 2006). Les études sur la fréquence avaient déjà mis en avant une plus grande aisance pour le traitement des mots fréquents par les patients Alzheimer; dans une tâche de dénomination d'images, les sujets atteints de la maladie d'Alzheimer et les sujets sains réagissent de manière similaire face à des mots fréquents, mais de grandes disparités apparaissent dès lors que les mots présentés sont non fréquents (Balota, Burgess, Cortese, Adams, 2002). Les mots fréquents seraient alors mieux intégrés dans le réseau sémantique; leur force de connexion tenant leur activation possible malgré la faiblesse cognitive.

L'analyse des résultats montre également un effet d'âge d'acquisition dans les productions des patients Alzheimer. Ceux-ci produisent des mots acquis plus tôt que les sujets sains en tâche sémantique. En accord avec les travaux antérieurs (Forbes-McKay et al., 2005), nos hypothèses sont vérifiées. Selon Steyvers \& Tenenbaum (2005), les mots qui sont acquis tôt s'inscriraient plus tôt dans 
un réseau sémantique où ils finiraient plus fortement interconnectés aux autres concepts que les mots acquis plus tard. Les mots acquis tôt permettraient une récupération plus facile, plus rapide et plus précise pour les patients. Les mots acquis tard, par contre, sont rares dans leurs productions. Les réseaux sémantiques des patients seraient donc plus faibles; seuls les mots inscrits dans des réseaux solides, encore pourvus de fortes connexions, seraient restés accessibles. Ces résultats sont compatibles avec la théorie de la rétrogenèse (Reisberg, 1989) selon laquelle les mécanismes dégénératifs renversent l'ordre d'acquisition : les mots acquis plus tôt dans l'existence semblent rester accessibles plus longtemps alors que les mots acquis plus tardivement ne sont pas ou plus difficilement récupérés par les patients. Ces résultats peuvent faire l'objet d'une application clinique dans le sens où ils permettent de prédire quels mots sont les plus robustes et lesquels sont les plus vulnérables à la maladie. Privilégier l'utilisation de mots acquis précocement avec le patient devrait permettre de maintenir la communication aussi longtemps que possible.

Enfin, les analyses de corrélations viennent valider notre troisième hypothèse : les effets de fréquence et d'âge d'acquisition sont significativement corrélés entre eux. Les mots acquis tôt dans la vie sont donc les mots fréquemment présentés à l'enfant. Toutefois, nos analyses ont également permis de soulever deux choses : (i) la corrélation entre fréquence et âge d'acquisition est quasi-identique entre les sujets contrôles et Alzheimer, laissant supposer qu'en tâche sémantique, les sujets Alzheimer emploient les mêmes stratégies de recherche que les sujets sains. Cette recherche, très probablement basée sur des critères sémantiques met les sujets Alzheimer en difficulté. Cette difficulté pourrait sous-tendre une désorganisation des réseaux sémantiques. (ii) La significativité de la corrélation entre les deux variables (fréquence et âge d'acquisition) s'atténue pour les sujets Alzheimer en tâche littérale par rapport aux sujets sains. La tâche littérale semble bien requérir une stratégie de recherche phonologique, basée sur des critères structuraux tels que l'ordre alphabétique, la longueur des mots, le voisinage phonologique, la complexité phonologique (Forbes-McKay et al, 2005; Lee, 2012 ; Gayraud \& Barkat-Defradas 2012), donnant moins de poids aux critères sémantiques des réseaux, et expliquant alors que les sujets Alzheimer aient des performances moins éloignées de celles des sujets contrôles dans cette tâche. Les sujets Alzheimer semblent bien souffrir d'une désorganisation des réseaux sémantiques. Cependant, en recherche phonologique, l'information semble rester plus accessible.

Ces résultats ne permettent pas de trancher en faveur de l'une ou l'autre des théories relatives au déficit sémantique observé. En effet, dans cette étude, nous avons pu remarquer que l'indiçage n'avait aucun effet sur les patients (e.g. Une patiente répète deux fois le même item ( "pamplemousse »), en précisant à chaque émission «je sais pas si c'est un fruit») (Warrington \& Shallice (1984), in Gainotti, Di Betta, Silveri,1996). Par ailleurs, les patients produisent un grand nombre d'erreurs de catégorie quand les sujets sains n'en produisent aucune. Cela supposerait plutôt la faiblesse des réseaux sémantiques et des connexions qui les constituent, qu'un problème d'accès (Rosser \& Hodges, 1994 ; Hodges \& Pattinson, 1995 ; Rascovsky, et al., 2007). Les paraphasies sémantiques, également conséquentes dans les productions des sujets Alzheimer, s'expliqueraient par les mêmes raisons (Murdoch, Chenery, Wilks, Boyle, 1987); tout comme les productions, par les patients, de catégories superordonnées («oiseaux») (tandis que les sujets sains produisent des hyponymes (« isard », " geai »)) (Tröster, Salmon, McCullough, Butters, 1989 ; Warrington \& Shallice (1984) in Gainotti et al., 1996). Enfin, l'évidence des effets de fréquence et d'âge d'acquisition, serait un fort argument en faveur de l'hypothèse de la théorie de la "rupture des réseaux sémantiques ». Pourtant, nous pouvons ajouter deux remarques soutenant davantage la théorie de l'accès déficitaire : (i) le déficit important des sujets atteints de la maladie d'Alzheimer en recherche sémantique, par rapport à la recherche littérale serait davantage dû à un problème d'accès, comme si l'information était toujours disponible mais inaccessible en recherche sémantique (Raskovsky et al., 2007); (ii) que des mots très rares et très peu fréquents (e.g. " raminagrobis »; " pédique ») apparaissent de temps en temps chez les patients va à l'encontre d'une désintégration des connaissances sémantiques.

Toutefois, si la fréquence et l'âge d'acquisition sont corrélés et si les deux semblent être caractéristiques du langage des personnes atteintes de la maladie d'Alzheimer, il semble préférable, au 
vu de la régression logistique, de privilégier les recherches sur l'âge d'acquisition. En effet, l'analyse de régression logistique, menée pour tenter de dissocier la fréquence et l'âge d'acquisition, nous permet de proposer que l'âge d'acquisition est la variable qui permet de prédire l'appartenance au groupe des sujets Alzheimer. Une personne qui produira des mots en moyenne acquis tôt, aura plus de chances de faire partie du groupe des sujets Alzheimer. L'âge d'acquisition semble être plus déterminant que la fréquence et nous permet alors de l'envisager, si ce n'est comme un facteur révélateur de la maladie d'Alzheimer, tout du moins comme une piste plus que sérieuse dans l'étude de cette démence. Nous supposons donc que l'âge d'acquisition représente un critère fiable, robuste et significatif dans la caractérisation du langage dans la maladie d'Alzheimer. C'est par ailleurs, ce que montre une étude de Forbes-McKay et al. (2005), dont les observations, reposant sur plusieurs caractéristiques du langage de patients atteints de la maladie d'Alzheimer (longueur des mots, fréquence, typicalité, âge d'acquisition), démontrent que l'âge d'acquisition est la variable la plus fiable, celle qui permet le mieux de prédire à quel groupe appartiennent les sujets de l'étude.

En dernier lieu, ajoutons que l'effet de génération trouvé dans les estimations d'âge d'acquisition, nous montre l'importance de bien apparier les échantillons entre eux et de bien analyser les productions de sujets âgés par des personnes âgées. C'est un choix d'outil méthodologique non négligeable. Si nous avons pu le vérifier sur nos propres données, les bases regroupant les âges d'acquisition ou les fréquences d'occurrence adaptées aux personnes âgées sont en pleine expansion (e.g. base « Freq Sub Jeune Senior 660 », Desrochers A., Bergeron M. 2000).

\section{Conclusion}

Au cours de ce travail, nous avons souhaité décrire au mieux les deux variables que sont la fréquence et l'âge d'acquisition, dans le processus de sélection lexicale des personnes atteintes de la maladie d'Alzheimer. En effet, la mémoire sémantique chez les sujets atteints de cette démence fait preuve de défaillances importantes, dont les répercussions pourraient être de bons indices de l'état d'avancement de la démence, mais également du fonctionnement de cette composante mnésique chez les patients. Effectivement, les travaux antérieurs ne sont pas d'accord sur les raisons d'un tel déficit langagier: est-ce une défaillance de l'accès à la mémoire (Pasquier et al., 1995) ou bien une faiblesse, voire une désintégration des connexions des réseaux sémantiques (Hodges \& Patterson, 1995) ? Nos travaux ne tranchent pas clairement en faveur de l'une ou l'autre de ces théories car, bien que montrant plus d'indices relatifs à une faiblesse des réseaux sémantiques, les sujets Alzheimer manifestent également des difficultés d'accès à la mémoire sémantique. Une hypothèse est que les deux théories ne soient pas mutuellement exclusives et que les deux phénomènes pourraient être à l'œuvre simultanément.

Notre travail nous permet cependant de poser la tâche de fluence verbale comme un outil efficace et révélateur des capacités lexicales des personnes atteintes de la maladie d'Alzheimer. Plus particulièrement et de manière importante, nous avons trouvé que les patients étaient très largement déficitaires dans les tâches impliquant une stratégie de recherche sémantique (i.e. tâches de fluence sémantique), alors qu'un exercice basé sur une recherche phonologique (i.e. tâche de fluence littérale), ne les met pas en aussi grande difficulté.

Une analyse tout d'abord numérique et globale des productions des sujets, fait ressortir que les patients produisent moins de mots et plus d'erreurs que les sujets sains. Au vu de nos résultats, nous pouvons alors dire que tous les effets observés ici ont apporté une différence entre les deux groupes de sujets. Autrement dit, les effets de fréquence et d'âge d'acquisition, connus pour faciliter la récupération lexicale chez les sujets contrôles étaient renforcés dans les productions des patients atteints de la maladie d'Alzheimer. Les personnes alors atteintes d'une telle démence produisent des mots plus fréquents et acquis plus tôt : des mots plus faciles d'accès. Il semble que les mots ne bénéficiant pas de connexions fortes et nombreuses dans le réseau sémantique, soient accessibles plus difficilement. 
Nous avons également mis en avant la corrélation entre ces deux variables en tâches sémantiques, postulant alors qu'elles jouent un rôle dans la récupération lexicale. Cependant, nous avons vu que ces deux variables étaient davantage corrélées dans les tâches requérant une stratégie sémantique : en stratégie littérale, c'est donc la logique phonologique qui est effective.

Enfin, les analyses de régression logistique nous indiquent que des deux variables : fréquence et âge d'acquisition, l'âge d'acquisition est significativement le meilleur prédicteur d'appartenance au groupe des sujets Alzheimer. L'âge d'acquisition nous semble donc être révélateur de la démence et s'inscrit véritablement comme un outil pertinent d'analyse et de description.

Les travaux dans le domaine doivent continuer: l'âge d'acquisition dans la maladie d'Alzheimer semble être un très bon outil de description mais surtout un révélateur des processus sémantiques. Il pourrait également être envisagé du point de vue de la rétrogenèse (Reisberg et al., 1999), posant que le lexique se désintègre dans un ordre inverse à celui de l'acquisition normale du langage.

\section{Références}

Adelman, J.S., Brown, G.D.A. \& Quesada, J.F. (2006). Contextual diversity, not word frequency, determines word-naming and lexical decision times. Psychological Science, 17(9), 814-823.

Appell, J., Kertesz, A. \& Fisman, M. (1982). A study of language functioning in Alzheimer patients. Brain and Language, 17, 73-91.

Balota, D.A., Burgess, G.C., Cortese, M.J. \& Adams, D.R. (2002). The word-frequency mirror effect in young, old and early-stage Alzheimer's disease : evidence for two processes in episodic recognition performance. Journal of Memory and Language, 46, 199-226.

Balota, D.A. \& Chumbley, J.I. (1985). The locus of word-frequency effect in the pronunciation task : Lexical access and/or production? Journal of Memory and Language, 24, 89-106.

Balota, D.A. \& Ferraro, F.R. (1996). Lexical, sublexical, and implicit memory processes in healthy young and healthy older adults and in individuals with dementia of the Alzheimer type. Neuropsychology, 10(1), 82-85.

Barkat-Defradas, M., Gayraud, F., Cadhilac, C. \& Lee, H.R. (2009). Pauses et hésitations dans le discours de patients Alzheimer et chez la personne âgée saine. Troisièmes journées de phonétique clinique (résumés), Aix-en-Provence, 20.

Barkat-Defradas, M., Martin, S., Duarte, L.-R., Brouillet, D. (2008). Les troubles de la parole dans la maladie d'Alzheimer, 27ème journée d'études sur la parole, Avignon.

Belliard, S., Péron, J., Lemoal, S., Golfier, V., Vanberten M. \& Vercelletto, M. (2005). in B.F, Michel, F., Verdureau \& P., Combet (Eds). Communication et démence, Marseille, Solal, 161-177.

Berr, C., Akbaraly, T.N., Nourashemi, F. \& Andrieu, S. (2007). Epidémiologie des démences. La Presse Médicale, 36(10-2), 1431-1441.

Blanpain, N. \& Chardon, O. INSEE. (2010). Première, [en ligne] http://www.insee.fr/fr/themes/document.asp?re f_i d=ip1320 \#inter3, 1320.

Brown, G.D.A. \& Watson, F.L. (1987). First-in, first-out: Word learning age and spoken word frequency as predictors of word familiarity and word naming latency. Memory and Cognition, 15(3), 208-216.

Bonin, P. (2003). Production verbale de mots. Approche cognitive, Bruxelles, De Boeck.

Campion, D. \& Hannequin D. (2002). La Maladie d'Alzheimer, Evreux, Flammarion.

Cardebat, D., Doyon, B., Puel, M., Goulet, P. \& Joanette, Y. (1990). Evocation lexicale formelle et sémantique chez des sujets normaux. Performances et dynamiques de production en fonction du sexe, de l'âge et du niveau d'étude. Acta Neurologica Belgica, 90, 207-217.

Caroll, J.B. \& White, M.N. (1973). Age-of-acquisition norms for 220 picturable nouns. Journal of Verbal Learning and Verbal Behaviour, 12. 563-576. 
Chalard, M., Bonin, P., Méot, A., Boyer, B., Fayol, M. (2003). Objective age-of-acquisition (AoA) norms for a set of 230 object names in French : Relationships with psycholinguistic variables, the English data from Morrison et al. (1997), and naming latencies. European Journal of Cognitive Psychology, 15(2), 209-245.

Desrochers, A. \& Bergeron, M. (2000). Valeurs de fréquence subjective et d'imagerie pour un échantillon de 1916 substantifs de la langue française. Revue Canadienne de Psychologie Expérimentale, 54(4), 274-325.

Donnet, A., Foncin, J.F. \& Habib, M. (1991). Démence et vieillissement cérébral : Evolution des concepts de l'Antiquité à nos jours. In M. Habib, Y. Joanette \& M. Puel (Eds). Démences et Syndromes démentiels, Approche neuropsychologique, Paris, Masson, 1-12.

Duarte, R.L., Jiménez, M., Syssau, A. \& Launay, M. (2004). Étude de la sensibilité d'une batterie d'épreuves sémantiques au vieillissement normal et à l'évolution de la maladie d'Alzheimer. Revue Européenne de Psychologie Appliquée, 54(3), 143-155.

Ellis, W.A. \& Lambon Ralph, M.A. (2000). Age of acquisition effects in adult lexical processing reflect loss of plasticity in maturing systems : insights from connectionists networks. Journal of Experimental Psychology: Learning, Memory and Cognition, 26(5), 1103-1123.

Ellis, W.-A. Morrison, C.-M. (1998). Real Age-of-Acquisition Effects in Lexical Retrieval. Journal of Experimental Psychology : Learning, Memory and cognition, 24(2), 515-523.

Ferrand, L., Bonin, P., Méot, A., Augustinova, M., New, B., Pallier, C. \& Brysbaert, M. (2008). Age-ofacquisition and subjective frequency estimates for all generally known monosyllabic French words and their relations with other psycholinguistic variables. Behavior Research Methods, 40(4), 1049-1054.

Folstein M.F., Folstein S.E. \& McHugh, P.R. (1975). Mini Mental State : A pratical method for grading the cognitive state of patients for the clinician. Journal of psychiatric Research,12, 189-198.

Forbes, K.E., Venneri, A. \& Shanks, A.W. (2002). Distinct patterns of spontaneous speech deterioration: an early predictor of Alzheimer's disease, Brain and Cognition, 48(2-3), 356-361.

Forbes-McKay, K.E., Ellis, A.W., Shanks, M.F. \& Venneri, A. (2005). The age of acquisition of words produced in a semantic fluency task can reliably differentiate normal from pathological age related cognitive decline. Neuropsychologia, 43, 1625-1632.

Gainotti, G., Di Betta, A.M. \& Silveri, M.C. (1996). The production of specific and generic associates of living and nonliving, high- and low-familiarity stimuli in Alzheimer's disease. Brain and Language, 54, 262-274.

Gayraud, F. \& Barkat-Defradas, M. (2012). Effet du voisinage phonologique sur l'accès lexical dans le discours spontané de patients Alzheimer. Actes de la conférence conjointe JEP-TALN-RECITAL 2012, Grenoble, Vol.1 $\therefore J E P, 369-376$.

Ghyselinck, M., Lewis, M.B. \& Brysbaert, M. (2004). Age of acquisition and the cumulative-frequency hypothesis : A review of the literature and a new multi-task investigation. Acta Psychologica, 115, 43-67.

Gilhooly, K.J. (1984) Word of age of acquisition and residence time in lexical memory as factors in word naming. Current Pyschological Research \& Reviews, Summer, 24-31.

Helmer, C., Pasquier, F. \& Dartigues, J.F. (2006). Épidémiologie de la maladie d'Alzheimer et des syndromes apparentés. $M / S$ : médecine sciences, 22(3), 288-296.

Hodges, J.R. \& Patterson, K. (1995). Is semantic memory consistently impaired early in the course of Alzheimer's disease? Neuroanatomical and diagnostic implications. Neuropsychologia, 33(4), 441-459.

INSEE Annuaire Statistique de la France. (2010) [en ligne] http://www.insee.fr/fr/themes/tableau.asp?reg_id=0\& ref_id=NATnon02147

INSEE Annuaire Statistique de la France. (2010). [en ligne], http://www.insee.fr/fr/themes/tableau.a sp?re g_id=0\&ref_id=NATnon0 229.

Jescheniak, J.D. \& Levelt, W.J.M. (1994). Word frequency effects in speech production: retrieval of syntactic information and of phonological form. Journal of Experimental Psychology : Learning, Memory, and Cognition, 20(4), 824-843. 
Kemper, S., Marquis, J. \& Thompson, M. (2001). Longitudinal change in language production : Effects of aging and dementia on grammatical complexity and propositional content. Psychology and aging, 16(4), 600-614.

Keulers, E., Brysbaert, M. \& New B. (2010). SUBTLEX-NL : A new mesure for dutch word frequency based on film subtitles. Behaviour Research Methods, 42(3), 643-650.

Kremin, H., Perrier, D., De Wilde, M., Dordain, M., Le Bayon, A., Gatignol, P., Rabine, C., Corbineau, M., Lehoux, E. \& Arabia, C. (2001). Factors predicting success in picture naming in Alzheimer's disease and primary progressive aphasia. Brain and Cognition, 46, 180-254.

Lee, H. (2012) Langage et Maladie d'Alzheimer : Analyse Multidimensionnelle d'un Discours Pathologique Thèse de doctorat en Sciences du Langage, Université Paul-Valéry, Montpellier 3.

Lee, H., Gayraud, F., Hirsh, F. \& Barkat-Defradas, M. (2011). Speech dysfluencies in normal and pathological aging: a comparison between Alzheimer patients and healthy elderly subjects. 17th International Congress of Phonetic Sciences (ICPhS), 1174-1177.

Levelt, W.J.M. (1999). Models of word production. Cognitive Sciences, 3(6), 223-233.

Lymperopoulou, O., Barry, C. \& Sakka, P. (2006). The effects of age of acquisition and word frequency on object naming accuracy in Alzheimer's disease. Annals of General Psychiatry, 5(1) : S76, 1.

Marczinski, C.A., Kertesz, A. (2006). Category and letter fluency in semantic dementia, primary progressive aphasia, and Alzheimer's disease. Brain and Language, 97, 258-265.

Melvold, J.L., Au, R., Obler, L.K. \& Albert, M.L. (1994). Language during aging and dementia. in M.L. Albert, \& J.E. Knoeffel (Eds). Clinical Neurology of Aging (2nde édition). New York : Oxford University Press.

McKhann, G., Drachman, D., Folstein, M., Katzman, R., Price, D. \& Stadlan, E.M. (1984). Clinical diagnosis of Alzheimer's disease : Report of the NINCDS-ADRDA Work Group under the auspices of Department of Health and Human Services Task Force on Alzheimer's Disease. Neurology, 34, 939-944.

Morrison, C.M., Hirsh, K.W., Chappell, T.D. \& Ellis, A.W. (2002). Age and age of acquisition : An evaluation of the cumulative frequency hypothesis. European Journal of Cognitive Psychology, 14(4), 435-459.

Morton, J. (1969). Interaction of information in word recognition. Psychological Review, 76(2), 165-178.

Murdoch, B.E., Chenery, H.J., Wilks, V. \& Boyle, R.S. (1987). Language disorders in dementia of the Alzheimer type. Brain and Language, 31, 122-137.

Nebes, R.D. (1989). Semantic memory in Alzheimer's disease. Psychological Bulletin, 106(3), 377-394.

New, B., Brysbaert, M., Veronis, J. \& Pallier, C. (2007). The use of film subtitles to estimate word frequencies. Applied Psycholinguistics, 28, 661-677.

New B., Pallier C., Ferrand L. \& Matos R. (2001). Une base de données lexicales du français contemporain sur internet: LEXIQUE. L'Année Psychologique, 101, 447-462, http://www.lexique.org

Ober, B.A. \& Shenaut, G.K. (1988). Lexical decision and priming in Alzheimer's disease. Neuropsychologia, 26(2), 273-286.

Oldfield R.C. \& Wingfield, A. (1965). Response latencies in naming objects. The Quaterly Journal of Experimental Psychology, 17(4), 273-281.

Pasquier, F., Lebert, F., Grymonprez, L. \& Petit, H. (1995). Verbal fluency in dementia of frontal lobe type and dementia of Alzheimer type. Journal of Neurology, Neurosurgery, and Psychiatry, 58, 81-84.

Puel, M., Démonet, J.F., Ousset, P.J. \& Rascol, O. (1991). La Maladie d'Alzheimer. In M. Habib, Y. Joanette, M. Puel, (Eds), Démences et syndromes démentiels, Approche neuropsychologique, Paris : Masson, 45-60.

Rascovsky, K., Salmon, D.P., Hansen, L., Thal, L.J. \& Galasko, D. (2007). Disparate letter and semantic category fluency deficits in autopsy-confirmed frontotemporal dementia and Alzheimer's disease. Neuropsychology, 21(1), 20-30.

Reisberg, B., Franssen, H.E., Hasan, M.S., Monteiro, I., Boksay, I., Souren, L.E.M., Kenowsky, S., Auer, S.R., Elahi, S. \& Kluger, A. (1999). Retrogenesis : Clinical, physiologic and pathologic mechanisms in brain aging, 
Alzheimer's and other dementing processes. European Archives of Psychiatry and Clinical Neuroscience, 249(3). 28-36.

Reisberg, B., Franssen, H.E., Souren, L.E.M., Auer, S.R., Akram, I. \& Kenowsky, S. (2002). Evidence and mechanisms of retrogenesis in Alzheimer's and other dementias : Management and treatment import. American Journal of Alzheimer's Disease and Other Dementias, 17(4). 202-212.

Rogers, H. \& Lasprilla, J.C.A. (2006). Retrogenesis theory in Alzheimer's disease : Evidence and clinical implications. Anales de Psicologia, 22(2), 260-266.

Rosser, A. \& Hodges, J.R. (1994). Initial letter and semantic category fluency in Alzheimer's disease, Huntington's disease and progressive supranuclear palsy. Journal of Neurology, Neurosurgery, and Psychiatry, 57, 1389-1394.

Sambuchi, N., Michel, B.F., Bastien, C. (2005). In B.F. Michel, F. Verdureau \& P. Combet, (Eds). Communication, langage oral et démence. Origine du manque du mot dans la maladie d'Alzheimer : Accès lexicale et mémoire sémantique. Communication et démence, Marseille : Solal, 63-82.

Segui, J., Mehler, J., Frauenfelder, U. \& Morton, J. (1982). The word frequency effect and lexical access. Neuropsychologia, 20(6), 615-627.

Ska, B. \& Duong, A. (2005). Communication, discours et démence. Psychologie et NeuroPsychiatrie du vieillissement, 3(2), 125-133

Steyvers, M., Tenenbaum, J.B. (2005). The large-scale structure of semantic networks: statistical analyses and a model of semantic growth. Cognitive Sciences, 29, 41-78.

Troster, A., Fields, J.A., Testa, J.A., Paul, R.H., Blanco, C.R., Hames, K.A., Salmon, D.P. \& Beatty, W.W. (1998). Cortical and subcortical influences on clustering and switching in the performance of verbal fluency tasks. Neuropsychologia, 36(4), 295-304.

Tulving, E. (1972) Episodic and semantic memory. in: E. Tulving \& W. Donaldson (Eds.), Organisation of Memory. Academic Press, New York, 381-403.

Tulving, E. (1976). Rôle de la mémoire sémantique dans le stockage et la récupération de l'information épisodique. Bulletin de Psychologie, Spécial Annuel, 19-25

Venneri, A., Forbes-McKay, K.E. \& Shanks, M.F. (2005). Impoverishment of spontaneous language and the prediction of Alzheimer's disease [Letter to the Editor], Brain, 128(4), E27.

Warrington, E.K. \& Shallice, T. (1984). Category specific semantic impairments, Brain, 103(3), 829-853. 